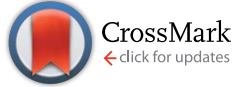

Cite this: RSC Adv., 2017, 7, 16109
Received 14th January 2017

Accepted 6th March 2017

DOI: 10.1039/c7ra00592j

rsc.li/rsc-advances

\section{Predicted structural evolution and detailed insight into configuration correlation, mechanical properties of silicon-boron binary compounds $\uparrow$}

\author{
Bo Zhang, ${ }^{\text {a }}$ Lailei $\mathrm{Wu}^{\mathrm{b}}$ and Zhihong $\mathrm{Li}^{\star a}$
}

\begin{abstract}
The crystal structures, phase stability, mechanical and electronic structures of silicon-boron binaries have been investigated systematically using first-principles of pseudopotential calculations. The calculated formation enthalpies of $\alpha-\mathrm{SiB}_{3}, \mathrm{SiB}_{6}$ and $\mathrm{SiB}_{n}$ follow the sequence: $\Delta H_{f}\left(\alpha-\mathrm{SiB}_{3}\right)>\Delta H_{f}\left(\mathrm{SiB}_{6}\right)>\Delta H_{f}\left(\mathrm{SiB}_{n}\right)$, which is in good agreement with the previous experimental results. Monoclinic $\mathrm{SiB}_{6}$ with $P 2_{1} / m$ symmetry and hexagonal $\mathrm{SiB}_{36}$ with a $\mathrm{B}_{80}$ unit (a ring linked by diametrically located $2 \times \mathrm{B}_{28}, 2 \times \mathrm{B}_{12}$ ) are suggested as the more energetically and mechanically favorable phases by our calculations. The predicted bulk modulus of $\mathrm{Si}-\mathrm{B}$ binaries is between 120 and $180 \mathrm{GPa}$, while there is a dramatic drop for two kinds of $\alpha-\mathrm{SiB}_{3}$ in the shear modulus from $130.3 \mathrm{GPa}\left(\mathrm{SiB}_{3}\right)$ to $71.1 \mathrm{GPa}\left(\mathrm{SiB}_{4}\right)$. We infer that the additional centered boron atoms, located long the spatial diagonal in $\mathrm{SiB}_{4}$, is responsible for the weakness along this direction. In addition to $\beta-\mathrm{SiB}_{3}$, the new proposed $P 2_{1} / m-\mathrm{SiB}_{6}$ and $R 3 m-\mathrm{SiB}_{6}$ is found to be semiconducting with $0.41 \mathrm{eV}$ indirect and $1.654 \mathrm{eV}$ direct band gap, respectively. There is no band gap provided by band structures of $\mathrm{SiB}_{4}$ and $\mathrm{SiB}_{36}$, as well as their DOS values are quite large at Fermi level, indicating they are energetically unstable under $\mathrm{OK}$ and $\mathrm{GPa}$.
\end{abstract}

\section{Introduction}

Boron-rich borides are classical refractory materials: lightweight, extremely hard, and exceptionally thermal stability. ${ }^{\mathbf{1 , 2}}$ Even more importantly, boron-rich compounds have attracted extensive attention for their high melting-points, semi-metallic/ metallic, ascending Seebeck coefficients and electrical conductivities with increasing temperature. ${ }^{3-5}$ Excellent electrical conductivity combined with weak thermal conductivity contributes to generate good thermoelectric properties in borides. In particular the silicon borides, possessing extremely higher chemical and thermal stabilities, have great potential for thermoelectric materials in extreme conditions (hightemperature, strong chemical corrosion, etc.). ${ }^{6,7}$ After reviewing the whole Si-B system, there are mainly three kinds of binary compounds, usually referred to as $\mathrm{SiB}_{3}, \mathrm{SiB}_{6}$ and $\mathrm{SiB}_{n}$. To be precise, " $\mathrm{SiB}_{3}$ " is the stoichiometry designation for $\mathrm{Si}-\mathrm{B}$ compounds ranging from $\mathrm{SiB}_{2.8}$ to $\mathrm{SiB}_{4} \cdot{ }^{8-15}$ This range is taken as an indication of a wide homogeneity phase region for a solid with the $\mathrm{SiB}_{3}$ structure. Another probable confusion should be

${ }^{a}$ Key Laboratory of Advanced Ceramics and Machining Technology of Ministry of Education, School of Materials Science and Engineering, Tianjin University, Tianjin 300072, China.E-mail: lizhihong@tju.edu.cn; Tel: +8622 27404260

${ }^{b}$ Key Laboratory of Metastable Materials Science and Technology, College of Material Science and Engineering, Yanshan University, Qinhuangdao 066004, China

$\dagger$ Electronic supplementary information (ESI) available. See DOI: 10.1039/c7ra00592j noticed that $\mathrm{SiB}_{3}$ here is more accurately $\alpha-\mathrm{SiB}_{3}$, since an orthorhombic phase, termed $\beta-\mathrm{SiB}_{3}$, has been obtained in gallium flux conditions at lower temperatures by J. R. Salvador et $a l .{ }^{16}$ Unless otherwise noted, $\alpha-\mathrm{SiB}_{3}$ is used in this paper to designate the phase with that kind of crystal structure and not to indicate a specific composition. Usually, $\alpha-\mathrm{SiB}_{3}$ is synthesized by melting a mixture of boron (5-10 weight\%) and silicon in a furnace, however, it is barely possible to obtain it purely, always being accompanied with $\mathrm{SiB}_{6}$ products. ${ }^{8-11,13,14} \alpha-\mathrm{SiB}_{3}$ has the same structure as boron carbide $\left(\mathrm{B}_{4} \mathrm{C}\right.$ or $\left.\mathrm{B}_{12} \mathrm{C}_{2}\right)$, and other borides belonging to a class of icosahedral boron-rich solids that includes $\alpha$-rhombohedral ( $\alpha$-rh) boron.,17,18 In 1956, Zhuravlev firstly found that $\mathrm{SiB}_{6}$ was a cubic phase through the X-ray structure analysis. ${ }^{19}$ Another different structure of $\mathrm{SiB}_{6}$ was determined by $\mathrm{M}$. Vlasse using single-crystal X-ray diffraction, ${ }^{20}$ it has a complex crystal structure with about 43 silicon atoms and 238 boron atoms in its orthorhombic unit cell, containing interconnected icosahedra, icosihexahedra, as well as several isolated boron and silicon atoms. Similar to $\alpha-\mathrm{SiB}_{3}$, the ratio of $\mathrm{B} / \mathrm{Si}=6 / 1$ is stoichiometry approximation for the $\mathrm{Si}$ B binaries ranging from $\mathrm{SiB}_{5.7}$ to $\mathrm{SiB}_{6.1}$ contributed by interchanging silicon and boron atoms within the multiple sites. ${ }^{17,20}$ $\mathrm{SiB}_{n}$ is obtained through the peritectic reaction: liquid $+\beta$ boron solid solution $=\mathrm{SiB}_{n}$ at $2293 \mathrm{~K},{ }^{6}$ and was first synthesized by Giese et al., known as $\operatorname{SiB}_{14} \cdot{ }^{21}$ However, the value width of $n$ has been a controversy so far that previous study claimed that $n$ ranges from 16 to $26 ;^{22}$ ten years later, T. B. Massalski et al. enlarged the $n$ values to the limits between 13.9 and $32.3 ;{ }^{23}$ after 
another decade, a wider value range of $n$ was introduced by Y. Imai et al. ${ }^{7}$ that the Si-rich solubility limit at $1873 \mathrm{~K}$ is 5.4 at\%, corresponding to $\mathrm{SiB}_{17.5}$, and the probable maximum value of $n$ reached up to 60 .

First-principles calculation is a valuable prediction approach to understand crystal parameters, thermodynamic properties and studying their structural transformations.,24,25 Band calculations of electronic densities of states and total energies of the $\mathrm{Si}-\mathrm{B}$ system have been attempted by Y. Imai ${ }^{7}$ using firstprinciples pseudopotential method within local density approximation. The theoretical achievements about $\mathrm{Si}-\mathrm{B}$ compounds were reviewed by B. Albert and H. Hillebrecht, ${ }^{25}$ crystal structures of this system and the structural features between the specific $\mathrm{Si}-\mathrm{B}$ compounds and their structurally related borides were summarized and sorted. $\beta$-Rhombohedral boron ( $\beta$-rh B) has been an attractive topic of calculation researches for decades. ${ }^{24-29}$ The crystal structure, thermodynamic stability, specific heat and electronic structure of $\beta$-rh $B$ were introduced by T. Ogitsu et al. ${ }^{\mathbf{2 4}}$ including experimental and theoretical calculation efforts. Shang et al. ${ }^{29}$ considered that $\beta$ rh B obtained several different atomic densities: 105-110 atoms per rhombohedral cell through examining the total energy of $\beta$ rh $\mathrm{B}$ as a function of atomic density. For the tetragonal configuration of boron, ${ }^{30-36}$ the crystal and electronic structures of $\alpha$-tetragonal ( $\alpha$-t or I-t) B ( $\mathrm{B}_{50}, 50$ atoms per cell) were investigated by Hayami and Otani ${ }^{31}$ from first-principles, while the other unstable $\beta$-tetragonal ( $\beta$-t or II-t) B is probably obtained through nonequilibrium growth based on the research of surface energy and crystal growth. ${ }^{35} \alpha$-rh $\mathrm{B}\left(\mathrm{B}_{12}\right)$, the significant basic icosahedral boron unit, has also been focused by researchers employing means of first-principles calculations, ${ }^{29,36-38}$ and it is shown that $\alpha$-rh $\mathrm{B}$ is a semiconductor with an indirect band gap of $1.70 \mathrm{eV}$ in the study of $\mathrm{D}$. Li et al. ${ }^{36}$ The majority of the research emphases were about the structures and properties of the elementary boron, and thermodynamic properties of the $\mathrm{Si}-\mathrm{B}$ compounds. However, to our knowledge, there are no comparative investigations on the mechanical properties and underlying relations between the phases of $\mathrm{Si}-\mathrm{B}$ system. Therefore, using means of first-principles calculations based on density functional theory, in this paper, we report the enthalpies of formation, elastic constants and electron structures of the $\mathrm{Si}-\mathrm{B}$ system and other related phases: $\alpha-\mathrm{SiB}_{3}\left(\mathrm{SiB}_{3}\right.$, $\left.\mathrm{SiB}_{4}\right), \beta-\mathrm{SiB}_{3}, \mathrm{SiB}_{6}, \mathrm{SiB}_{36}$. The relevant data of $\alpha$-rhombohedral boron ( $\alpha$-rh B), $\beta$-rhombohedral boron ( $\beta$-rh B), $\alpha$-tetragonal boron ( $\alpha$-t B), and $\gamma-B_{28}$ (high pressure phases) are also available in $\mathrm{ESI} \dagger$ for comparison and comprehensive understanding.

\section{Computational details}

First-principles calculations were performed using CASTEP code based on Density Functional Theory (DFT). ${ }^{39}$ The exchange and correlation functions were treated by generalized gradient approximation with the parameterization by Perdew-BurkeErnzerhof (GGA-PBE). ${ }^{\mathbf{4 0 4 1}}$ The electronic configurations of each element were $2 \mathrm{~s}^{2} 2 \mathrm{p}^{1}$ for $\mathrm{B}$ and $3 \mathrm{~s}^{2} 3 \mathrm{p}^{2}$ for $\mathrm{Si}$, respectively. The mass of $\mathrm{B}$ atom is 10.81 and $\mathrm{Si}$ atom is 28.085. The oxidation state of any atom is 0 , and none temperature factors were set on atoms. The convergence of calculations was initially checked by a $5 \times 10 \times 10 \mathrm{k}$-point and cutoff energy of $310 \mathrm{eV}$. The ground state and elastic constants $\left(C_{i j}\right)$ of all considered $\mathrm{Si}-\mathrm{B}$ phases are then calculated for each fully relaxed structure. Bulk and shear moduli were estimated based on Voight-Reuss-Hill (VRH) approximation. ${ }^{42}$ Elastic moduli $(E)$ and Poisson's ratio $(\nu)$ were obtained based on the relationship: $E=9 B G /(3 B+G), \nu=(3 B-$ $2 G) /(6 B+2 G)$. The universal elastic anisotropy index $\left(A^{\mathrm{U}}\right)^{43}$ is obtained from the $B$ and $G$ of Voigt and Reuss with the equation: $A^{\mathrm{U}}=5 G_{\mathrm{V}} / G_{\mathrm{R}}+B_{\mathrm{V}} / B_{\mathrm{R}}-6$.

\section{Results and discussions}

\subsection{Structural stability}

Mechanical stability, a necessary condition for a stable phase to exist in experiments, was firstly checked for all of the $\mathrm{Si}-\mathrm{B}$ system crystals according to the Born-Huang criterion. ${ }^{\mathbf{4 4 , 4 5}}$ If all the individual elastic moduli of a specific phase are satisfied, the mechanical stability criteria indicate its stability. The calculated elastic constants of $\mathrm{Si}-\mathrm{B}$ system phases are listed in Table 1 , and it can be found that $\alpha-\mathrm{SiB}_{3}\left(\mathrm{SiB}_{3}, \mathrm{SiB}_{4}\right), \beta-\mathrm{SiB}_{3}$ and $\mathrm{SiB}_{36}$ satisfy the Born-Huang criterion, indicating their mechanical stabilities. However, cubic $P m \overline{3} m-\mathrm{SiB}_{6}$ (ref. 19) $\left(C_{44}=-4.13 \mathrm{GPa}\right)$ is mechanically unstable. To get a further understanding of the structural configuration of $\mathrm{SiB}_{6}$, we have employed the in-house developed CALYPSO ${ }^{\mathbf{4 6 , 4 7}}$ structure prediction methodology to conduct the structure search for $\mathrm{SiB}_{6}$. Our search identified 20 stable structures: $\mathrm{SiB}_{6}-01 \_01$, $\mathrm{SiB}_{6}-02 \_01, \ldots, \mathrm{SiB}_{6}-19 \_01, \mathrm{SiB}_{6}-20 \_01$. Monoclinic $P 2_{1} / m$-type (space group number: 11 ) $\mathrm{SiB}_{6}-01 \_01$ with the lowest energy is founded to be the most energetically favorable among the 20 configurations, calculated energy of these 20 structures are available in the ESI. $\dagger$ Hexagonal $R 3 m$-type (space group number: 160) $\mathrm{SiB}_{6}-08 \_01$ is also considered in this study because of its special structure (it will be discussed in the following sections). For convenience, $\mathrm{SiB}_{6}-01 \_01$ denotes as $\mathrm{SiB}_{6}$ and $\mathrm{SiB}_{6}-08 \_01$ is abbreviated as $\mathrm{SiB}_{6}-81$. According to Table 1, monoclinic $P 2_{1} / m-\mathrm{SiB}_{6}$ and hexagonal $R 3 m-\mathrm{SiB}_{6}-81$ are qualified for mechanical stability. To furtherly prove the reliability of the new suggested structure of $\mathrm{SiB}_{6}$, the XRD pattern was calculated. As shown in Fig. 1, it can be found that our theoretical results are in generally accordance with the experimental XRD data $^{\mathbf{1 7 , 4 8 , 4 9}}$ in consideration of only isolated data provided from the previous work, ${ }^{48}$ which the XRD peaks of $\mathrm{Si}, \mathrm{SiB}_{3}$ and other $\mathrm{Si}-\mathrm{B}$ compounds have been eliminated from based on available reports. ${ }^{17,49}$

To verify the thermodynamic stability of the considered phases in the $\mathrm{Si}-\mathrm{B}$ system, the formation enthalpies $\left(\Delta H_{\mathrm{f}}\right)$ are calculated as listed in Table 2 and compared with available experimental results. ${ }^{22,50}$ The formation enthalpy of $\mathrm{Si}_{x} \mathrm{~B}_{y}$ phase can be defined as

$$
\Delta H_{\mathrm{f}}=E_{\text {total }}\left(\mathrm{Si}_{x} \mathrm{~B}_{y}\right)-\left(x E_{\text {total }}(\mathrm{Si})+y E_{\text {total }}(\mathrm{B})\right)
$$

where $E_{\text {total }}\left(\mathrm{Si}_{x} \mathrm{~B}_{y}\right)$ is the total energy of $\mathrm{Si}_{x} \mathrm{~B}_{y}$ at equilibrium lattice constants; $E_{\text {total }}(\mathrm{Si})$ and $E_{\text {total }}(\mathrm{B})$ are the calculated total energy of $\mathrm{Si}(-101.901 \mathrm{eV})$ and $\mathrm{B}(-70.488 \mathrm{eV}), x$ and $y(x+y=1)$ 
Table 1 Calculated elastic constants $C_{i j}$ (in $\mathrm{GPa}$ ) of considered $\mathrm{Si}-\mathrm{B}$ phases

\begin{tabular}{|c|c|c|c|c|c|c|c|c|c|c|}
\hline Phase & S.G. & $C_{11}$ & $C_{22}$ & $C_{33}$ & $C_{44}$ & $C_{55}$ & $C_{66}$ & $C_{12}$ & $C_{13}$ & $C_{14}$ \\
\hline $\mathrm{SiB}_{3}$ & $R \overline{3} m$ & 326.0 & & 349.3 & 144.0 & & & 86.3 & 92.1 & -2.47 \\
\hline $\mathrm{SiB}_{4}$ & $R \overline{3} m$ & 259.0 & & 373.5 & 51.7 & & & 125.8 & 104.3 & -37.9 \\
\hline \multirow[t]{2}{*}{$\mathrm{SiB}_{6}$} & $P m \overline{3} m$ & 402.6 & & & -4.13 & & & 19.31 & & \\
\hline & $P 2_{1} / m$ & $\begin{array}{l}373.6 \\
29.5\left(C_{23}\right)\end{array}$ & 351.6 & $\begin{array}{l}432.5 \\
16.0\left(C_{15}\right)\end{array}$ & 127.2 & $\begin{array}{l}176.4 \\
-5.8\left(C_{25}\right)\end{array}$ & 174.8 & $\begin{array}{l}101.5 \\
-23.7\left(C_{35}\right)\end{array}$ & 101.4 & $-20.5\left(C_{46}\right)$ \\
\hline $\mathrm{SiB}_{6}-81$ & $R 3 m$ & 187.8 & & 196.1 & 117.3 & & & 74.0 & 93.3 & 74.9 \\
\hline $\mathrm{SiB}_{36}$ & $R 3 m$ & 419.3 & & 431.5 & 146.8 & & & 72.9 & 75.6 & 36.7 \\
\hline$\beta-\mathrm{SiB}_{3}$ & Imma & $\begin{array}{l}288.8 \\
72.6\left(C_{23}\right)\end{array}$ & 369.6 & 357.4 & 123.3 & 134.6 & 130.8 & 51.5 & 64.3 & \\
\hline
\end{tabular}



Fig. 1 Calculated $\mathrm{X}$-ray diffraction pattern of $P 2_{1} / m$-SiB 6 compared with experimental data.

are the atomic fractions of $\mathrm{Si}$ and $\mathrm{B}$, respectively. According to Table 2, it can be observed that our calculated formation enthalpies of $\mathrm{Si}-\mathrm{B}$ phases generally agree with the experimental results in the previous reports. ${ }^{22,48}$ Taking $\alpha-\mathrm{SiB}_{3}\left(\mathrm{SiB}_{3}, \mathrm{SiB}_{4}\right)$, $\mathrm{SiB}_{6}$ and $\mathrm{SiB}_{36}\left(\mathrm{SiB}_{n}\right)$ for comparison, $\mathrm{SiB}_{36}\left(\mathrm{SiB}_{n}\right)$ has the most negative $\Delta H_{\mathrm{f}}(-2.883 \mathrm{eV}$ per atom) and the formation enthalpies of the three kinds of compounds follow the sequence of $\Delta H_{\mathrm{f}}(\alpha-$ $\left.\mathrm{SiB}_{3}\right)>\Delta H_{\mathrm{f}}\left(\mathrm{SiB}_{6}\right)>\Delta H_{\mathrm{f}}\left(\mathrm{SiB}_{n}\right)$. Consequently, our calculated results are in good agreement with the previous experimental data, ${ }^{51}$ that $\mathrm{SiB}_{n}$ has the highest stable temperature (about 2200
$\mathrm{K}$ ) and the lowest (about $1400 \mathrm{~K}$ ) is for $\alpha-\mathrm{SiB}_{3}$. For $\mathrm{SiB}_{3}$ and $\mathrm{SiB}_{4}$, they present little difference in the formation enthalpies, since these two binaries share similar structure belonging to $\alpha-\mathrm{SiB}_{3}$ phase. It can also be furtherly determined that $P 2_{1} / m$ - $\mathrm{SiB}_{6}$ is more energetically favorable than $P m \overline{3} m-\mathrm{SiB}_{6}$ and $\mathrm{SiB}_{6}-81$, since $\Delta H_{\mathrm{f}}$ of the former one is less than the latter ones. $\alpha-\mathrm{SiB}_{3}\left(\mathrm{SiB}_{3}\right.$, $\mathrm{SiB}_{4}$ ) and $\gamma-\mathrm{B}_{28}$ is structurally related to $\alpha$-rh $\mathrm{B}$, from Table 2 and $\mathrm{ESI}, \dagger$ it can be found that $\alpha$-rh B is the most thermodynamic stable phase of those related structures and it is naturally interpreted as foreign atoms ( $\mathrm{Si}$ atoms) disturbing the original charge distribution, thus more energy being needed to maintain equilibrium of the crystal system. However, the $\Delta H_{\mathrm{f}}$ of $\mathrm{SiB}_{36}$ whose structure is based on the framework of $\beta$-rh B is much more negative than $\beta$-rh B. Different from the previous condition, introducing $\mathrm{Si}$ atoms to the complicated polyatomic $\beta$-rh B crystal is helpful to reduce internal energy of the structure. The rigorous synthesis and stability conditions, that $\beta-\mathrm{SiB}_{3}$ needs producing from a lower temperature reaction $\left(1000-850{ }^{\circ} \mathrm{C}\right)$ in metallic flux to permit the total bypass of the compound $\alpha$ $\mathrm{SiB}_{3},{ }^{16}$ indicate the highest $\Delta H_{\mathrm{f}}$ of $\beta-\mathrm{SiB}_{3}$ in Table 2.

The calculated structural parameters and Wyckoff positions of different crystals, after full stress and position relaxation, are shown in Table 3 and ESI. $\dagger$ In general, our results are in good accordance with previous theoretical and experimental results as shown in Table $3 .^{9,11,16,19,52}$

As shown in Fig. 2, $\mathrm{B}_{12}$ icosahedra, as the single primary unit of $\alpha$-rh B, is an indispensable structural element for most of the $\mathrm{Si}-\mathrm{B}$ compounds. It can be found that $\mathrm{SiB}_{3}$ (Fig. 2a) and $\mathrm{SiB}_{4}$ (Fig. 2b) are established with $\mathrm{B}_{12}$ icosahedra as the framework. The $\mathrm{B}_{12}$ units are situated on the corners of a rhombohedron

Table 2 Calculated formation enthalpies of $\mathrm{Si}-\mathrm{B}$ system compared with available experimental results

\begin{tabular}{|c|c|c|c|c|}
\hline Phase & S.G. & $\begin{array}{l}\Delta H_{\mathrm{f}}(\mathrm{eV} \\
\text { per atom })\end{array}$ & $\begin{array}{l}\Delta H_{\mathrm{f}}(\mathrm{kJ} \\
\text { per mol per atom })\end{array}$ & $\begin{array}{l}\Delta H_{\mathrm{f}} \text { (Exp.) } \\
\text { (kJ per mol per atom) }\end{array}$ \\
\hline $\mathrm{SiB}_{3}$ & $\operatorname{Hex} .(R \overline{3} m)$ & -1.196 & -115.449 & -109.4 (ref. 48) \\
\hline \multirow{2}{*}{$\mathrm{SiB}_{6}$} & Cub. $(P m \overline{3} m)$ & -1.218 & -117.486 & \multirow{3}{*}{$-148.3^{48}$} \\
\hline & Mon. $\left(P 2_{1} / m\right)$ & -1.267 & -122.233 & \\
\hline $\mathrm{SiB}_{6}-81$ & Hex. $(R 3 m)$ & -1.248 & -120.458 & \\
\hline $\mathrm{SiB}_{36}$ & Hex. $(R \overline{3} m)$ & -2.883 & -278.161 & \\
\hline$\beta-\mathrm{SiB}_{3}$ & Ort.(Imma) & -0.0699 & -6.746 & \\
\hline
\end{tabular}


Table 3 Calculated and experimental lattice parameters of considered Si-B phases

\begin{tabular}{|c|c|c|c|c|c|c|c|}
\hline & Structure & \multicolumn{6}{|c|}{ Lattice parameter $(\AA)$} \\
\hline $\mathrm{SiB}_{3}$ & $\operatorname{Hex} .(R \overline{3} m)$ & 6.319 & 6.319 & 12.71 & 6.32 & 6.32 & 12.71 (ref. 11) \\
\hline $\mathrm{SiB}_{4}$ & $\operatorname{Hex} .(R \overline{3} m)$ & 6.319 & 6.319 & 12.71 & 6.33 & 6.33 & 12.74 (ref. 9) \\
\hline $\mathrm{SiB}_{6}-81$ & $\operatorname{Hex} .(R 3 m)$ & 5.85 & 5.85 & 7.48 & & & \\
\hline $\mathrm{SiB}_{36}$ & $\operatorname{Hex} .(R \overline{3} m)$ & 11.01 & 11.01 & 23.90 & 10.98 & 10.98 & 23.86 (ref. 51) \\
\hline$\beta-\mathrm{SiB}_{3}$ & Ort.(Imma) & 8.39 & 12.57 & 6.21 & 8.39 & 12.57 & 6.21 (ref. 16) \\
\hline
\end{tabular}

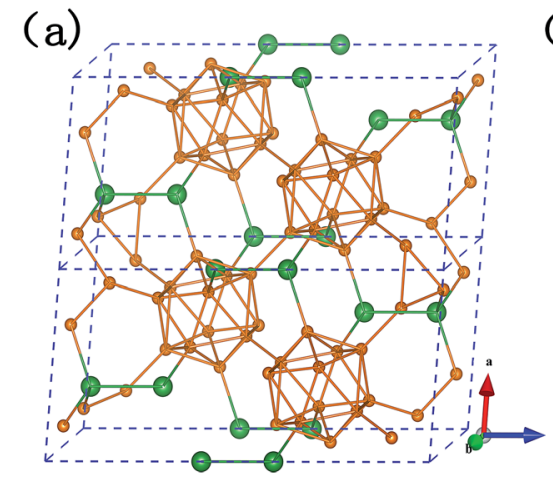

(d)

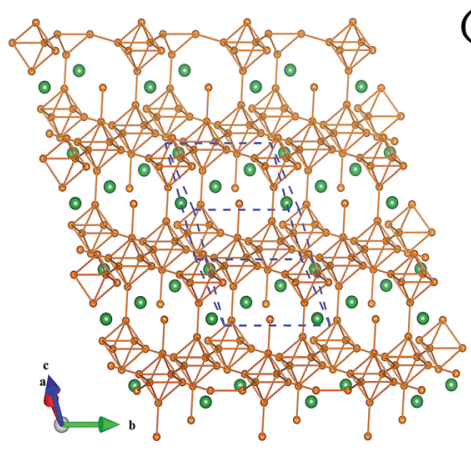

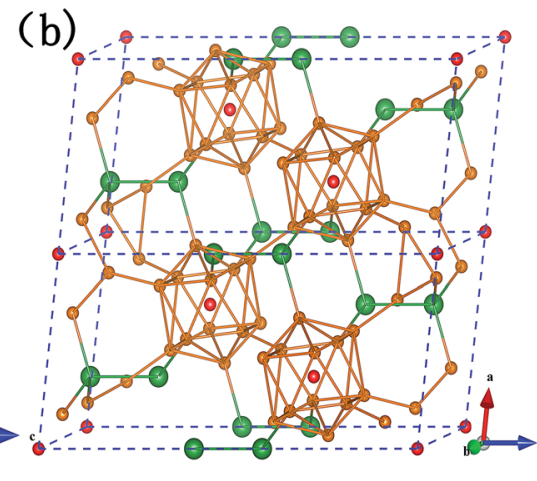

(e)

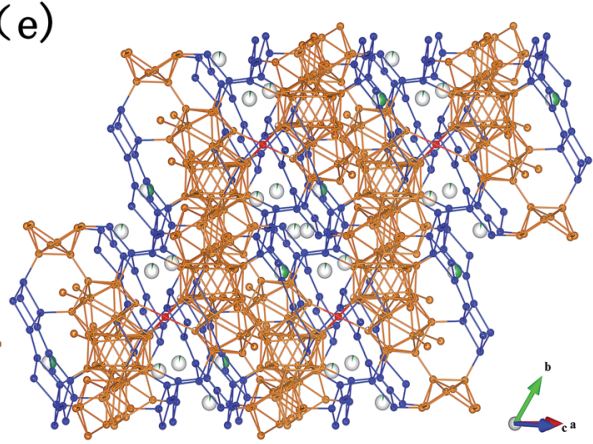

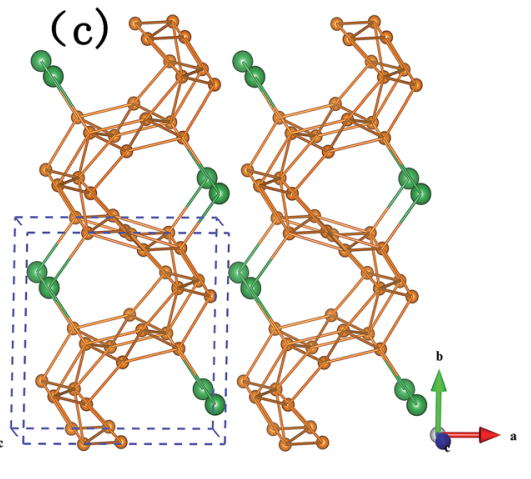

(f)

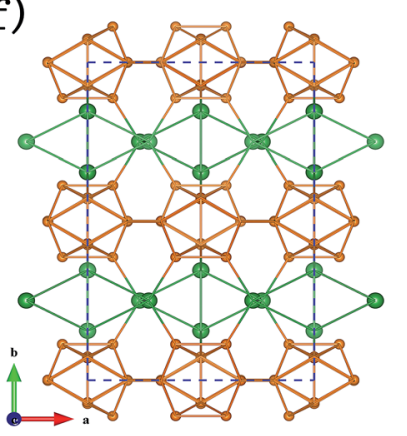

Fig. 2 Crystal structures of six $\mathrm{Si}-\mathrm{B}$ compounds after full stress and position relaxation. (a) $\mathrm{SiB}_{3} ;$ (b) $\mathrm{SiB}_{4} ;$ (c) $P 2_{1} / m-\mathrm{SiB}_{6}$; (d) $\mathrm{SiB}_{6}-81$; (e) $\mathrm{SiB}_{36}$; (f) $\beta-\mathrm{SiB}_{3}$. Boron and silicon atoms are marked as brown and green, respectively; boron atoms in special positions of their structures were marked as red in (d) (e), respectively; boron bilayers were displayed in blue color in (e) $\mathrm{SiB}_{36}$.

just as in $\alpha$-rh B (two $18 \mathrm{~h}$ sites in hexagonal arrangement), Si atoms are located on the spatial diagonal of the rhombohedron and link three icosahedra each of one layer (6c site), as a result, the remaining valence electron of $\mathrm{Si}$ atom contribute to bonding a pair of close $\mathrm{Si}$ atoms to form $\mathrm{a} \mathrm{Si}_{2}$ group. Thus, the $2 \mathrm{e} 3 \mathrm{c}$ boron bonds (B-B distance: $1.67 \AA$ ) in $\alpha$-rh B are replaced by $2 \mathrm{e} 2 \mathrm{c}$ silicon bonds ( $\mathrm{Si}-\mathrm{Si}$ distance: $2.39 \AA$ for $\mathrm{SiB}_{3}$ and $2.29 \AA$ for $\mathrm{SiB}_{4}$ ), causing a distinct reduction of the $c / a$ ratio $(12.56 / 4.908$ for $\alpha$-rh $\mathrm{B}$ in hexagonal arrangement). Overall, it is the partial incorporation, which results from silicon atoms going into the polar position of the icosahedron, that leads to the high silicon content and the relatively large lattice parameters.

The remarkable difference between $\mathrm{SiB}_{3}$ and $\mathrm{SiB}_{4}$ is that there is an additional $\mathrm{B}$ atom (3a site) in $\mathrm{SiB}_{4}$ lattice located in the center of each icosahedron, accordingly, which induce slight variations of the bond lengths of inter- and intra-icosahedral boron atoms. However, such little change did not affect the lattice parameters in our calculated results (as shown in Table 3). It also can be explained that in $\alpha-\mathrm{SiB}_{3}$, some $\mathrm{Si}$ atoms are randomly distributed in the $\mathrm{B}_{12}$ cages resulting in very distorted icosahedra, ${ }^{18}$ according to different situation of inside Si atoms of $\mathrm{B}_{12}$-cages in these two $\alpha-\mathrm{SiB}_{3}$ phases. In contrast, $\mathrm{Si}$ atoms in $\beta-\mathrm{SiB}_{3}$ are rigorously excluded from the cages (as shown in Fig. 2f), thus resulting in more regular icosahedra. It was even considered as the first $\mathrm{Si}-\mathrm{B}$ binary phase that is fully crystallographically ordered without any $\mathrm{Si} / \mathrm{B}$ mixed sites or disorder. ${ }^{16}$

The structures of two $\mathrm{SiB}_{6}$ compounds are quite different. In Fig. 2d, the hexagonal crystal $\left(R 3 m-\mathrm{SiB}_{6}-81\right)$ belongs to layered- 
like structure based on the $\mathrm{B}_{6}$ octahedron unit composed of eight boron atoms, and with silicon atoms located in interstitial spaces; however, configuration of the monoclinic one $\left(P 2_{1} / \mathrm{m}\right.$ $\mathrm{SiB}_{6}$ ) is built on zig-zag networks bonded by boron atoms in Fig. 2c.

Comparing the Si-B binaries (Fig. $2 \mathrm{a}-\mathrm{f}$ ), it can be noticed that the structures bonded with boron atoms become more and more complex along with the increasing content of boron. For example, $\mathrm{B}_{28}$ triply fused icosahedra (three condensed icosahedra in Fig. 2e) were formed in $\mathrm{SiB}_{36}$ by 28 boron atoms in addition to $\mathrm{B}_{12}$ icosahedra-the only polyhedron in $\alpha-\mathrm{SiB}_{3}$. Si atoms partially occupy the interstitial sites between the polyhedrons in $\mathrm{SiB}_{36}$ instead of being located in the $6 \mathrm{c}$ sites completely in $\alpha-\mathrm{SiB}_{3}$. With regard to the structural influence on the $\mathrm{B}-\mathrm{B}$ bond lengths of inter- and intra-icosahedra, we find that the distances are all beyond $1.85 \AA$ in the $\mathrm{B}_{12}$ units of two $\alpha$ $\mathrm{SiB}_{3}$ structures, while they reduce to around $1.70 \AA$ in $\mathrm{SiB}_{36}$.

As the last two phases in the boron-rich end of the $\mathrm{Si}-\mathrm{B}$ phase diagram, $\mathrm{SiB}_{36}$ and $\beta$-rh $\mathrm{B}$ have a similar formation based on $\mathrm{B}_{28}$ triply fused icosahedra as the boron framework. Differing to previous structures of $\mathrm{SiB}_{36}$ (ref. 25 and 53) those could be analogized as $\beta$-rh B framework with interstitial sites between polyhedrons and $\mathrm{B}$ atoms in icosahedra partly occupied by $\mathrm{Si}$ atoms, however, in this work there is a new found boron $\mathrm{B}_{6}$ unit comprised with 6 boron atoms. To be specific, as highlighted by blue atoms in Fig. $2 \mathrm{e}$, two quadrilateral- $\mathrm{B}_{4}$ connect with each other by a shared $\mathrm{B}-\mathrm{B}$ bond $\left(\mathrm{B}_{7}-\mathrm{B}_{9}\right.$, bond length: $\left.1.79 \AA\right)$ producing two included angle: $121.62^{\circ}$ and $115.64^{\circ}$. The linked $\mathrm{B}_{6}$ units together with $\mathrm{B}$ atoms of $\mathrm{B}_{28}$ units form a layer being vertical to the spatial diagonal. There are four kinds of boron positions in $\mathrm{a}_{6}$ unit, $\mathrm{B}_{1}$ : bonded with two neighbor $\mathrm{B}$ atoms of its own $\mathrm{B}_{6}$ unit and $\mathrm{B}$ atom of the neighbor $\mathrm{B}_{6}$ unit in the same layer; $B_{2}$ : bonded with one $B$ atom of $B_{28}$ unit, one of the neighbor parallel layer, in addition to two intra- $\mathrm{B}_{6}$ unit $\mathrm{B}-\mathrm{B}$ bonds; $\mathrm{B}_{7}$ : three intra- $\mathrm{B}_{6}$ unit $\mathrm{B}-\mathrm{B}$ bonds and a $\mathrm{B}-\mathrm{B}$ bond with a $B$ atom in a icosahedron; $B_{9}$ : similar to $B_{7}$ except the bond with icosahedral atom, however, linked with a $\mathrm{B}$ atom of $\mathrm{B}_{28}$ unit. The lengths of $\mathrm{B}-\mathrm{B}$ bond intra- $\mathrm{B}_{6}$ unit are $\mathrm{B}_{1}-\mathrm{B}_{2}: 1.71 \AA \mathrm{B}_{2}-\mathrm{B}_{7}$ : $1.79 \AA, \mathrm{B}_{1}-\mathrm{B}_{9}: 1.82 \AA, \mathrm{B}_{7}-\mathrm{B}_{9}: 1.79 \AA$, respectively. The bond lengths of between intra- and inter- $B_{6}$ unit are layer-layer: $B_{2}-B_{2}$ $=1.78 \AA$, intra- $\mathrm{B}_{6}$ with $\mathrm{B}_{28}: \mathrm{B}_{9}-\mathrm{B}_{10}=1.69 \AA$ and $\mathrm{B}_{2}-\mathrm{B}_{3}=1.73 \AA$, intra- $\mathrm{B}_{6}$ with $\mathrm{B}_{12}: \mathrm{B}_{7}-\mathrm{B}_{5}=1.67 \AA$.

From Fig. 2e and 3, we can find that two layers constituted with $\mathrm{B}_{80}$ units $\left(\mathrm{B}_{80}\right.$, a ring linked by diametrically located $2 \times$ $\mathrm{B}_{28}, 2 \times \mathrm{B}_{12}$ ) are connected by two neighbor parallel $\mathrm{B}_{6}$ layers, in addition to be covalently bound through junctional atom $\mathrm{B}_{15}$ (highlighted as red atoms in Fig. 2e). Therefore, it can be considered as $\mathrm{B}_{80}$ layers and $\mathrm{B}_{6}$ layers being stacked vertically to the spatial diagonal make the framework of $\mathrm{SiB}_{36}$, and $\mathrm{Si}$ atoms are partially located at interstitial sites in the space surrounded by the joint $\mathrm{B}_{6}$ layers.

\subsection{Elastic properties}

The calculated individual elastic constants within the strainstress method are listed in Table 1 for considered Si-B system crystals. In general, all of the mechanically stable phases show

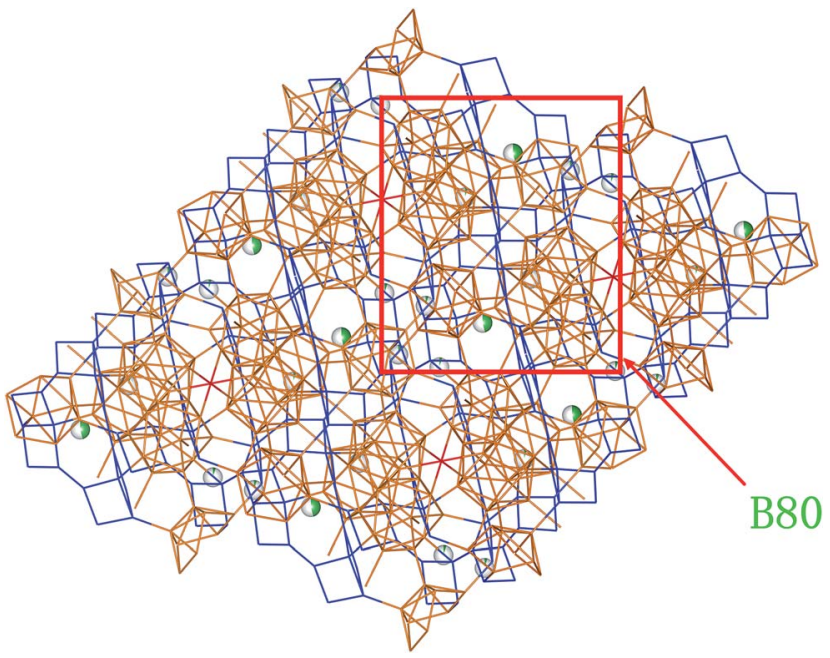

Fig. $3 \mathrm{~B}_{80}$ unit (a ring linked by diametrically located $2 \times \mathrm{B}_{28}$ and $2 \times$ $\left.\mathrm{B}_{12}\right)$ in $\mathrm{SiB}_{36}$.

the considerably higher values of $C_{11}, C_{22}$ and $C_{33}$ than that of $C_{44}$, indicating relatively higher incompressibility along $a$-, $b$ and $c$-axis. Furthermore, among all the individual elastic constants, $C_{33}(432.5 \mathrm{GPa})$ of $P 2_{1} / m-\mathrm{SiB}_{6}$ is the largest one suggesting its specially low compressibility along $c$-axis. Additionally, the $C_{11}$ value of $\mathrm{SiB}_{36}$ reaches up to $419.3 \mathrm{GPa}$ confirming its outstanding incompressibility along $a$-axis; meanwhile, its $C_{33}$ is $431.5 \mathrm{GPa}$, the second highest value, predicting the excellent elastic properties of $\mathrm{SiB}_{36}$.

To further demonstrate the elastic properties of Si-B crystals, bulk modulus, shear modulus, Young's modulus, Poisson's ratio and universal elastic anisotropy index $\left(A^{\mathrm{U}}\right)$ were estimated from the calculated individual elastic constants (as shown in Table 4), the previous available results are also listed for comparison and verifying the reliability of our calculation (as shown in ESI $\dagger$ ). In general, our calculated results are in reasonable accordance with the reported data, however, it should not be ignored that the bulk moduli $(181.3 \mathrm{GPa})$ of $\beta$-rh B is $11 \%$ less than the theoretical result $(203.5 \mathrm{GPa})$ predicted by Masago, et $a l^{37}$ The different approximation methods may induce the deviation between the two considered values, since local-density approximation (LDA) was employed in ref. 37 while our $a b$ initio calculation adopted GGA as the approximation method. In consideration of first-principles calculation for elastic properties (elastic constant, bulk/shear moduli, etc.), LDA results are probably larger than the actual values, on the other hand, GGA method is likely to complete tasks with underestimation, which can also explain the fact that our results are relatively smaller than the experimental data. According to Table 4, the bulk modulus (183.5 GPa) of $\mathrm{SiB}_{36}$ is the largest $B$, while $\mathrm{SiB}_{6}$ has the largest $G(153.8 \mathrm{GPa})$ and $E$ $(358.8 \mathrm{GPa})$. The intensity parameters of the other phases are just slightly lower, also indicating their comparative hardness. However, it is unexpected that the shear moduli of $\mathrm{SiB}_{4}$ drops to $55.4 \mathrm{GPa}$, for its structure is particularly similar to $\mathrm{SiB}_{3}(G=$ 129.8 GPa) and their bulk modulus are very close (172.1 GPa for $\mathrm{SiB}_{4}, 171.2 \mathrm{GPa}$ for $\mathrm{SiB}_{3}$ ). Comparing the two structures in Fig. 2, 
Table 4 The calculated bulk modulus ( $B, B_{\vee}$ and $B_{\mathrm{R}}$ in $\left.G P a\right)$, shear modulus ( $G, G_{\vee}$ and $G_{R}$ in $\left.G P a\right)$, Young's modulus ( $E$ in $\left.G P a\right)$, Poisson's ratio $(\nu)$, Pugh's ration $(B / G)$ and universal elastic anisotropy $\left(A^{\cup}\right)$ of $\mathrm{Si}-\mathrm{B}$ system

\begin{tabular}{|c|c|c|c|c|c|c|c|c|c|c|c|}
\hline Phase & S.G. & $B$ & $B_{\mathrm{V}}$ & $B_{\mathrm{R}}$ & $G$ & $G_{\mathrm{V}}$ & $G_{\mathrm{R}}$ & $E$ & $\nu$ & $\mathrm{B} / \mathrm{G}$ & $A^{\mathrm{U}}$ \\
\hline $\mathrm{SiB}_{3}$ & $R \overline{3} m$ & 171.2 & 171.4 & 171.1 & 129.8 & 130.3 & 129.3 & 310.8 & 0.20 & 1.32 & 0.041 \\
\hline $\mathrm{SiB}_{4}$ & $R \overline{3} m$ & 172.1 & 173.4 & 170.7 & 55.4 & 71.1 & 39.6 & 150.0 & 0.35 & 3.11 & 4.00 \\
\hline $\mathrm{SiB}_{6}-81$ & $R 3 m$ & 121.1 & 121.4 & 120.8 & 64.3 & 79.0 & 49.5 & 163.8 & 0.27 & 1.88 & 2.99 \\
\hline $\mathrm{SiB}_{36}$ & $R 3 m$ & 183.5 & 186.1 & 180.9 & 152.4 & 153.2 & 151.6 & 358.1 & 0.17 & 1.20 & 0.082 \\
\hline$\beta-\mathrm{SiB}_{3}$ & Imma & 153.6 & 154.7 & 152.5 & 132.5 & 132.9 & 132.0 & 308.6 & 0.17 & 1.16 & 0.048 \\
\hline
\end{tabular}

we infer that the additional boron atoms in $\mathrm{SiB}_{4}$ lattice, contrast to $\mathrm{SiB}_{3}$, are just right located long the spatial diagonal so as to disturb the original electronic division and impair the original bonding strength, that finally induce the conspicuous weakness along this direction which be responsible for the shear performance of crystals.

It could not be ignored that not only the elastic performances of $P 2_{1} / m-\mathrm{SiB}_{6}$ and $R 3 m-\mathrm{SiB}_{6}-81$ are quite different, but also the elastic modulus of $R 3 m-\mathrm{SiB}_{6}-81$ are much lower than the other $\mathrm{Si}-\mathrm{B}$ compounds. After analyzing the structures in Fig. $2 \mathrm{c}$ and $\mathrm{d}$ and 4 , we can conclude that the hexagonal crystal $\left(R 3 m-\mathrm{SiB}_{6}{ }^{-}\right.$ 81) belongs to layered-like structure based on the $B_{6}$ octahedron unit composed of eight boron atoms, and with silicon atoms located in interstitial spaces; however, configuration of the monoclinic one $\left(P 2_{1} / m-\mathrm{SiB}_{6}\right)$ seems to be more complex with relative more covalent networks not only between boron atoms but also silicon ones involved, which avoiding layers' sliding in the lattice structure of $R 3 m-\mathrm{SiB}_{6}-81$ and providing $P 2_{1} / m-\mathrm{SiB}_{6}$ stronger elastic properties.

The above few fluctuations can't impede us to find these general trends that both the bulk and shear modulus decreased gradually with the increasing $\mathrm{Si}$ concentration. Those are possibly induced by that $\mathrm{B}-\mathrm{B}$ bond distance ranges are lengthened from 1.67-1.92 $\AA$ in monatomic boron to $1.84-2.04 \AA$ so as to weaken the bond strength, in addition to the balance of electron distribution is broken and the new $\mathrm{Si}-\mathrm{Si}, \mathrm{Si}-\mathrm{B}$ bonds are inferior to B-B bonds in hardness, contributed by the incorporation of Si atoms into the stable structure bonded by $\mathrm{B}$ atoms.
The ratio between the shear and bulk modulus $(B / G)$ has been proposed by $\mathrm{Pugh}^{54}$ to predict brittle or ductile behavior of materials. According to the Pugh criterion, a high $B / G$ value indicates a tendency for ductility, while a low $B / G$ value is associated with brittleness. From Table $4, \mathrm{SiB}_{4}(2.80), \mathrm{SiB}_{6}-81$ (1.88) are recognized with $B / G$ value larger than the critical value (1.75) suggesting their ductile behaviors, while the other phases are expected to perform brittleness. The elastic anisotropy index $\left(A^{\mathrm{U}}\right)$ represents a universal measure to quantify the single crystal elastic anisotropy. As shown in Table $4, \mathrm{SiB}_{3}$ is the relatively most isotropic phase, with the lowest $A^{\mathrm{U}}$ value (0.041) in the whole $\mathrm{Si}-\mathrm{B}$ system, contrarily, $\mathrm{SiB}_{4}\left(A^{\mathrm{U}}=4.00\right)$ is predicted as most anisotropy crystal.

\subsection{Electronic properties}

The total and partial density of states (DOS) for the Si-B crystals are displayed in Fig. 5. Analyzing all the DOS patterns, we found that there are three phases: $P 2_{1} / m-\mathrm{SiB}_{6}, R 3 m-\mathrm{SiB}_{6}-81$ and $\beta-\mathrm{SiB}_{3}$ showing the semiconducting features. Incorporated with the calculated band structures of those 3 compounds (shown in Fig. 6), we collected the indirect band gaps for $P 2_{1} / m$-SiB $B_{6}$ and $\beta$ $\mathrm{SiB}_{3}$ with energy values (eV): 0.41 and 1.461; direct band gaps for $R 3 m-\mathrm{SiB}_{6}-81(1.654 \mathrm{eV})$, respectively. The calculated result of $\beta$ $\mathrm{SiB}_{3}$ is in good accordance with the previously work ${ }^{\mathbf{1 6}}$ which showed an indirect band gap of $\sim 1.5 \mathrm{eV}$ from theoretical calculations and $2.0 \mathrm{eV}$ optically determined, in addition, our calculated band gaps of $\alpha$-rh B $(1.533 \mathrm{eV})$ and $\gamma-\mathrm{B}_{28}(1.580 \mathrm{eV})$ in $\mathrm{ESI} \uparrow$ are in good agreement with previously theoretical results:



Fig. 4 Crystal structure of hexagonal $\mathrm{SiB}_{6}-81$. (a), (b) and (c) are along $a-,, b$ - and c-axis, respectively. 

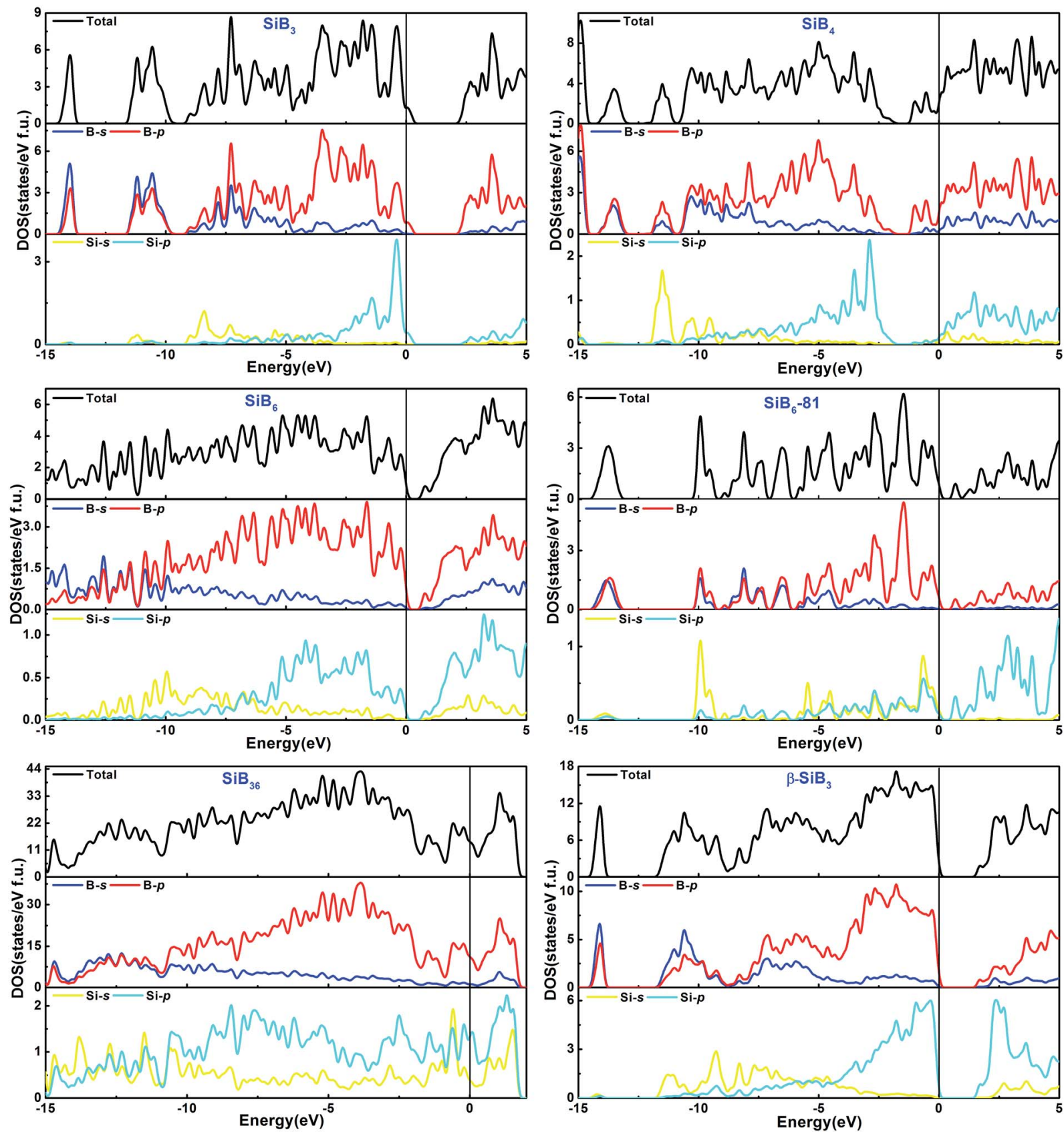

Fig. 5 Calculated total and partial density of states of phases in Si-B system. Fermi energy level is indicated by a vertical line.

$1.54 \mathrm{eV}$ (ref. 25) and $1.7 \mathrm{eV},{ }^{55}$ therefore our calculation of electronic properties is dependable. The estimated band gap of $\mathrm{SiB}_{6}$ by Feigelson and Kingery is about $1.2 \mathrm{eV}$ (ref. 56) and our calculated value is $0.41 \mathrm{eV}$. Since the general tendency is allowed that the generalized gradient approximation gives narrower band gap than the observed one, the estimated value seems to be reasonable. The temperature dependence of the electrical conductivity of $\mathrm{SiB}_{3}$ was measured in ref. 56 and it was concluded that $\mathrm{SiB}_{3}$ is a semiconductor with the energy gap of $1.2 \mathrm{eV}$, which does not agree to the present work. We infer that the increase in electric conductivity with the temperature observed by Feigelson and Kingery ${ }^{56}$ is due to the rising hopping probability of the localized state at higher temperature, which is possibly induced by disordered arrangement of the atoms, but due to the semiconducting band conduction. $R 3 m-\mathrm{SiB}_{6}-81$ has a wide band gap of $1.654 \mathrm{eV}$, this new theoretically suggested material might be useful as a refractory n-type semiconductor capable of operating in extreme environments, so the results reported herein warrant future investigations of synthesizing this Si-B compound.

Since it is widely accepted that the DFT-GGA approximation probably underestimates the band gap of solids, ${ }^{24,32}$ DFT-LDA approximation was also applied to study the electronic structures and verify the GGA calculation results. The LDA 

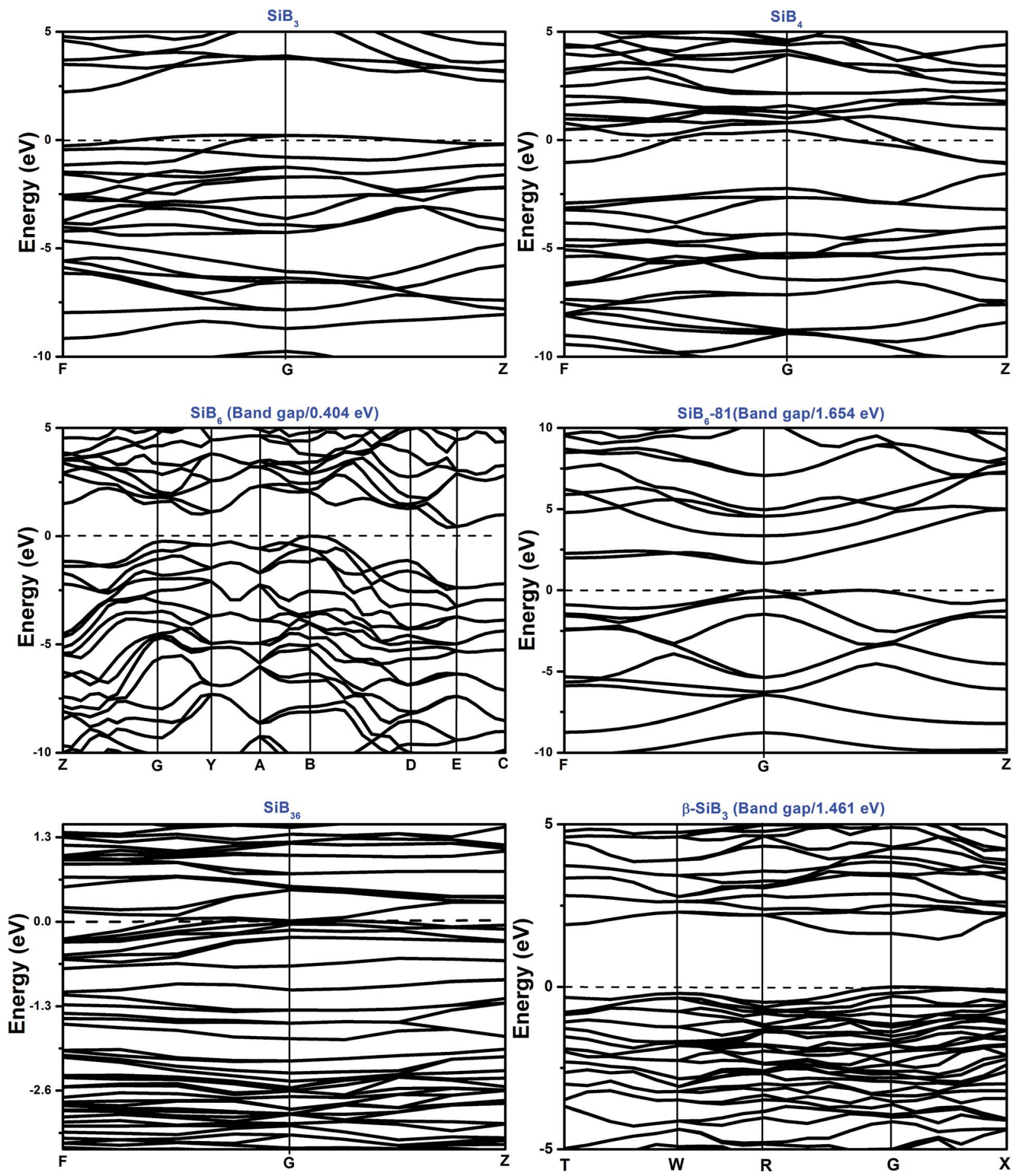

Fig. 6 Calculated band structures of $\mathrm{Si}-\mathrm{B}$ phases. Fermi energy level is indicated by a dotted line.

calculation results indicate that the band gaps are $0.371 \mathrm{eV}$, $1.621 \mathrm{eV}$ and $1.502 \mathrm{eV}$ for $\mathrm{SiB}_{6}, \mathrm{SiB}_{6}-81$ and $\beta-\mathrm{SiB}_{3}$, respectively. Those reasonable differences between GGA and LDA calculations solidify our conclusions in electronic properties research. The DOS and band structures results from DFT-LDA calculations are available in ESI. $\dagger$

There is no band gap provided by band structures of $\mathrm{SiB}_{4}$ and $\mathrm{SiB}_{36}$ in Fig. 6, as well as their DOS values are relatively large at Fermi level, indicating they are energetically unstable under $0 \mathrm{~K}$ and $\mathrm{GPa}$. It can be found that there is a wide overlap between B- $s$ state and $\mathrm{B}-p$ state of $\mathrm{SiB}_{3}, \mathrm{SiB}_{4}, \mathrm{SiB}_{6}, \mathrm{SiB}_{6}-81, \mathrm{SiB}_{36}$ and $\beta-\mathrm{SiB}_{3}$ from $-14 \mathrm{eV}$ to $-5 \mathrm{eV}$, respectively, demonstrating the strong $\mathrm{sp}^{3}$ hybridization of covalent bonds, which mainly accounts for their relatively good performances in bulk modulus. It is interesting to notice that for $\mathrm{SiB}_{3}$ and $\mathrm{SiB}_{4}$, there are two sharp peaks in both sides of the Fermi level and DOS between the peaks are not $0 \mathrm{eV}$, which implying pseudogap exists in both of these two phases. Those pseudogap valleys show stronger 
covalent interaction in the structures. Moreover, there is a general feature for the $\mathrm{Si}-\mathrm{B}$ binaries that below the Fermi level, the valence bands were dominated by B states with both B$s$ and B- $p$ states at lower band while B- $p$ states at higher band. Nevertheless, as shown in Fig. 5, the contributions to the total DOS of Si-B compounds from the Si states are not significant because $\mathrm{Si}$ atoms donate electrons to B-B network to stabilize the structure, for example, about 0.96 electron transformed from one $\mathrm{Si}$ atom to $\mathrm{B}-\mathrm{B}$ network for $\mathrm{SiB}_{6}$ by analyzing the atomic Mulliken overlap populations.

In addition to the calculated values of two indirect band gaps and one direct band gap, in the band structures of these six $\mathrm{Si}-\mathrm{B}$ binaries (shown in Fig. 6), it can also be noticed that the distributions of band structures of $P 2_{1} / m-\mathrm{SiB}_{6}, \mathrm{SiB}_{36}$ and $\beta-\mathrm{SiB}_{3}$ are more concentrated than those of the other three phases; and each energy band at different points fluctuates less drastically. Those details account for that electron localizations of the energy bands of $\mathrm{SiB}_{6}, \mathrm{SiB}_{36}$ and $\beta-\mathrm{SiB}_{3}$ are more intensive and effective mass of an electron is relatively larger; in contrast, energy bands of $\mathrm{SiB}_{3}, \mathrm{SiB}_{4}$ and $R 3 m-\mathrm{SiB}_{6}-81$ display more electron nonlocality.

\section{Conclusions}

We systematically investigated the crystal structures, phase stability, mechanical properties and electronic structures of silicon-boron system by using first-principles calculations of density functional theory. Previously suggested phase: $P m \overline{3} m$ $\mathrm{SiB}_{6}$ is found to be mechanically unstable, and the new structure of $P 2_{1} / m-\mathrm{SiB}_{6}$ is proposed in this paper. Another new structure of $\mathrm{SiB}_{36}$ is suggested, in which two layers constituted with $\mathrm{B}_{80}$ unit are connected by two neighbor parallel $\mathrm{B}_{6}$ layers, in addition to be covalently bound through junctional atom $B_{15}$ while Si atoms are partially located at interstitial sites in the space surrounded by the joint $\mathrm{B}_{6}$ layers. We find the general trends that both the bulk and shear modulus decreased gradually with the increasing Si concentration, because the introduced $\mathrm{Si}-\mathrm{Si}$ and $\mathrm{Si}-\mathrm{B}$ bonds are weaker than the original $\mathrm{B}-\mathrm{B}$ network. Although the elastic properties of $\beta-\mathrm{SiB}_{3}$ are slightly inferior to $\alpha-\mathrm{SiB}_{3}$, its wide band gap (1.461 eV) makes $\beta-\mathrm{SiB}_{3}$ a potential p-type semiconductor. In addition to $\beta$-SiB ${ }_{3}, P 2_{1} / m$ $\mathrm{SiB}_{6}$ is found to be with an indirect band gap of $0.41 \mathrm{eV}$; while $R 3 m-\mathrm{SiB}_{6}-81$ has a direct band gap of $1.654 \mathrm{eV}$, this new theoretically suggested material might be useful as a refractory ntype semiconductor capable of operating in extreme environments that deserving more efforts in research of this $\mathrm{Si}-\mathrm{B}$ compound.

\section{References}

1 R. Riedel, Adv. Mater., 1994, 6, 549-560.

2 Mechanical properties and their relation to microstructure, D. Sherman and D. Brandon, Wiley-VCH Verlag $\mathrm{GmbH}$, Weinheim, Germany, 2000.

3 C. Wood, Mater. Res. Soc. Symp. Proc., 1987, 97, 335-346.

4 J.-M. Darolles, T. Lepetre and J.-M. Dusseau, Phys. Status Solidi A, 1980, 58, K71-K75.
5 M. Mukaida, T. Tsunoda, M. Ueda and Y. Imai, Proc. 19th Int. Conf. Thermoelectrics, 2000, p. 255.

6 J. Wu, W. Ma, D. Tang, B. Jia, B. Yang, D. Liu and Y. Dai, Procedia Eng., 2012, 31, 297-301.

7 Y. Imai, M. Mukaida, M. Ueda and A. Watanabe, J. Alloys Compd., 2002, 347, 244-251.

8 C. Brosset and B. Magnusson, Nature, 1960, 187, 54-55.

9 V. I. Matkovich, Acta Crystallogr., 1960, 13, 679-680.

10 H. F. Rizzo and L. R. Bidwell, J. Am. Ceram. Soc., 1960, 43, 550-552.

11 B. Magnusson and C. Brosset, Acta Chem. Scand., 1962, 16, 449-455.

12 On refractory boron compounds crystallizing in $\mathrm{B}_{4} \mathrm{C}$-type structures, ed. T. Lundstrom and H. Bolmgren, Jpn. J. Appl. Phys. Ser., 1994, vol. 10, pp. 1-4.

13 E. Colton, J. Am. Chem. Soc., 1960, 82, 1002.

14 C. F. Cline and D. E. Sands, Nature, 1960, 185, 456.

15 R. Tremblay and R. Angers, Ceram. Int., 1989, 15, 73-78.

16 J. R. Salvador, D. Bilc, S. D. Mahanti and M. G. Kanatzidis, Angew. Chem., 2003, 115, 1973-1976.

17 T. L. Aselage, J. Mater. Res., 1998, 13, 1786-1794.

18 T. L. Aselage and D. R. Tallant, Phys. Rev. B: Condens. Matter Mater. Phys., 1998, 57, 2675-2678.

19 N. N. Zhuravlev, Kristallografiya, 1956, 1, 666-668.

20 M. Vlasse, G. A. Slack, M. Garbauskas, J. S. Kasper and J. C. Viala, J. Solid State Chem., 1986, 63, 31-45.

21 R. F. Giese, J. Economy and V. I. Matkovich, Z. Kristallogr., 1965, 122, 144-147.

22 B. Armas, G. Male, D. Salanoubat, C. Chatillon and M. Allibert, J. Less-Common Met., 1981, 82, 245-254.

23 T. B. Massalski, H. Okamoto, P. R. Subramanian and L. Kacprzak, Binary alloy phases diagrams, ASM, Materials Park, OH, 2nd edn, 1990.

24 T. Ogitsu, E. Schwegler and G. Galli, Chem. Rev., 2013, 113, 3425-3449.

25 B. Albert and H. Hillebrecht, Angew. Chem., Int. Ed., 2009, 48, 8640-8668.

26 B. Callmer, Acta Crystallogr., Sect. B: Struct. Crystallogr. Cryst. Chem., 1977, 33, 1951-1954.

27 D. L. V. K. Prasad, M. M. Balakrishnarajan and E. D. Jemmis, Phys. Rev. B: Condens. Matter Mater. Phys., 2005, 72, 195102.

28 T. Ogitsu, F. Gygi, J. Reed and Y. Motome, J. Am. Chem. Soc., 2009, 131, 1903-1909.

29 S. Shang, Y. Wang, R. Arroyave and Z. K. Liu, Phys. Rev. B: Condens. Matter Mater. Phys., 2007, 75, 092101.

30 S. Lee, D. Bylander, S. Kim and L. Kleinman, Phys. Rev. B: Condens. Matter Mater. Phys., 1992, 45, 3248-3251.

31 W. Hayami and S. Otani, J. Solid State Chem., 2010, 183, 1521-1528.

32 S. Aydin and M. Simsek, J. Alloys Compd., 2011, 509, 52195229.

33 J. L. Hoard, R. E. Hughes and D. E. Sands, J. Am. Chem. Soc., 1958, 80, 4507-4515.

34 G. Will and K. Ploog, Nature, 1974, 251, 406-408.

35 W. Hayami and S. Otani, J. Phys. Chem. C, 2007, 111, 1039410397. 
36 D. Li, Y. N. Xu and W. Y. Ching, Phys. Rev. B: Condens. Matter Mater. Phys., 1992, 45, 5895-5905.

37 A. Masago, K. Shirai and H. Katayama-Yoshida, Phys. Rev. B: Condens. Matter Mater. Phys., 2006, 73, 104102.

38 M. A. White, A. B. Cerqueira, C. A. Whitman, M. B. Johnson and T. Ogitsu, Angew. Chem., Int. Ed., 2015, 54, 3626-3629.

39 P. J. D. L. M. D Segall, M. J Probert, C. J Pickard, P. J Hasnip, S. J Clark and M. C Payne, J. Phys.: Condens. Matter, 2002, 14, 2717-2744.

40 J. P. Perdew, J. A. Chevary, S. H. Vosko, K. A. Jackson, M. R. Pederson, D. J. Singh and C. Fiolhais, Phys. Rev. B: Condens. Matter Mater. Phys., 1992, 46, 6671-6687.

41 J. P. Perdew, K. Burke and M. Ernzerhof, Phys. Rev. Lett., 1996, 77, 3865-3868.

42 R. Hill, Proc. Phys. Soc., 1952, 65, 349-354.

43 S. I. Ranganathan and M. Ostoja-Starzewski, Phys. Rev. Lett., 2008, 101, 055504.

44 M. Born and K. Huang, Dynamical theory of crystal lattices, Oxford University Press, Oxford, 1954.

45 J. F. Nye, Physical properties of crystals, Oxford University Press, Oxford, 1985.

46 Y. Wang, J. Lv, L. Zhu and Y. Ma, Phys. Rev. B: Condens. Matter Mater. Phys., 2010, 82, 094116.
47 Y. Wang, J. Lv, L. Zhu and Y. Ma, Comput. Phys. Commun., 2012, 183, 2063-2070.

48 C. F. Cline, J. Electrochem. Soc., 1959, 106, 322-325.

49 JCPDS pattern \#35-777, International Centre for Diffraction Data, Newton Square, PA.

50 A. I. Zaitsev and A. A. Kodentsov, J. Phase Equilib., 2001, 22, 126-135.

51 R. W. Olesinski and G. J. Abbaschian, Bull. Alloy Phase Diagrams, 1984, 5, 478-484.

52 B. G. Arabey, Izv. Akad. Nauk SSSR, Neorg. Mater., 1979, 15, 1589-1592.

53 J. Roger, V. Babizhetskyy, J.-F. Halet and R. Guérin, J. Solid State Chem., 2004, 177, 4167-4174.

54 S. F. Pugh, Philos. Mag., 1954, 45(7), 823-843.

55 E. Zarechnaya, L. Dubrovinsky, N. Dubrovinskaia, Y. Filinchuk, D. Chernyshov, V. Dmitriev, N. Miyajima, A. El Goresy, H. Braun, S. Van Smaalen, I. Kantor, A. Kantor, V. Prakapenka, M. Hanfland, A. Mikhaylushkin, I. Abrikosov and S. Simak, Phys. Rev. Lett., 2009, 102, 185501. 56 R. S. Feigelson and W. D. Kingery, Ceram. Bull., 1963, 42, 688. 